\title{
Signet-ring Cell Carcinoma of the Gallbladder Complicated by Pulmonary Tumor Thrombotic Microangiopathy
}

\author{
Yoshinori Ohno ${ }^{1}$, Teru Kumagi ${ }^{1,2}$, Taira Kuroda ${ }^{1}$, Mitsuhito Koizumi ${ }^{1}$, Nobuaki Azemoto ${ }^{1}$, \\ Hirofumi Yamanishi ${ }^{1}$, Mayu Oda ${ }^{3}$, Masashi Hirooka ${ }^{1}$, Masanori Abe ${ }^{1}$, Yoshio Ikeda ${ }^{1}$, \\ Bunzo Matsuura ${ }^{1}$, Morikazu Onji ${ }^{1}$, Yoshiko Soga ${ }^{4}$, Yousuke Mizuno ${ }^{4}$, \\ Atsurou Sugita ${ }^{4}$ and Yoichi Hiasa ${ }^{1}$
}

\begin{abstract}
Biliary drainage was performed in a 71-year-old man with obstructive jaundice of unknown origin; however, he died due to acute pulmonary failure. At autopsy, proliferation of adenocarcinoma cells was observed in the gallbladder mucosa transitioning from isolated signet-ring cell carcinoma (SRCC) to the subserosa and bile ducts without growth toward the gallbladder lumen. Furthermore, fibrocellular intimal proliferation, tumor emboli and organized thrombi were observed in the small pulmonary arteries. The final diagnosis was gallbladder carcinoma complicated by SRCC associated pulmonary tumor thrombotic microangiopathy (PTTM). PTTM may present as rapidly progressive dyspnea, and a high level of clinical suspicion is required to make the differential diagnosis.
\end{abstract}

Key words: signet-ring cell carcinoma, gallbladder, pulmonary tumor thrombotic microangiopathy

(Intern Med 53: 1125-1129, 2014)

(DOI: 10.2169/internalmedicine.53.1939)

\section{Introduction}

Gallbladder carcinoma (GBC) is known to be an aggressive neoplasm, thus providing early detection and radical surgery is mandatory in order to achieve a better prognosis (1). However, approximately $50 \%$ of patients with GBC present with the disease at advanced stage, with locoregional spread and lymph node metastasis (2). Adenocarcinoma is the most appreciated histological type of GBC, while signetring cell carcinoma (SRCC) is extremely rare (2). In general, the aggressive behavior of SRCC is evidenced by its infiltration of the surrounding stroma, broad dissemination and a high tendency to produce peritoneal metastases in the gastrointestinal tract (3). In addition, in patients with pulmonary tumor thrombotic microangiopathy (PTTM), histological examinations reveal medial and intimal hypertrophy, intimal fibrosis and fibrinoid necrosis of the internal elastic lamina of small arteries and arterioles $(4,5)$. Pulmonary hypertension and cor pulmonale induced by tumor emboli causing PTTM are rare and extremely difficult to diagnose prior to death (6). We herein present a case of SRCC of the gallbladder complicated by PTTM.

\section{Case Report}

A 71-year-old Japanese man was admitted to our hospital due to right upper abdominal pain and jaundice in May 2011. He had a history of total gastrectomy for gastric malignant lymphoma in 1986. On admission, he was jaundiced, and a mass was palpable in the right upper abdomen with tenderness. Laboratory tests showed abnormalities in the levels of hepatobiliary enzymes and bilirubin: AST $=380 \mathrm{U} / \mathrm{L}$, $\mathrm{ALT}=252 \mathrm{U} / \mathrm{L}, \mathrm{ALP}=2,591 \mathrm{U} / \mathrm{L}, \mathrm{GGT}=1,087 \mathrm{U} / \mathrm{L}$ and total bilirubin $=8.6 \mathrm{mg} / \mathrm{dL}$. The levels of tumor markers were also elevated: carcinoembryonic antigen $=529 \mathrm{ng} / \mathrm{mL}$ and carbo-

\footnotetext{
${ }^{1}$ Department of Gastroenterology and Metabology, Ehime University Graduate School of Medicine, Japan, ${ }^{2}$ Department of Community Medicine, Ehime University Graduate School of Medicine, Japan, ${ }^{3}$ Residency Program, Ehime University Hospital, Japan and ${ }^{4}$ Pathology Division, Ehime University Hospital, Japan

Received for publication October 17, 2013; Accepted for publication December 17, 2013

Correspondence to Dr. Teru Kumagi, terukuma@m.ehime-u.ac.jp
} 

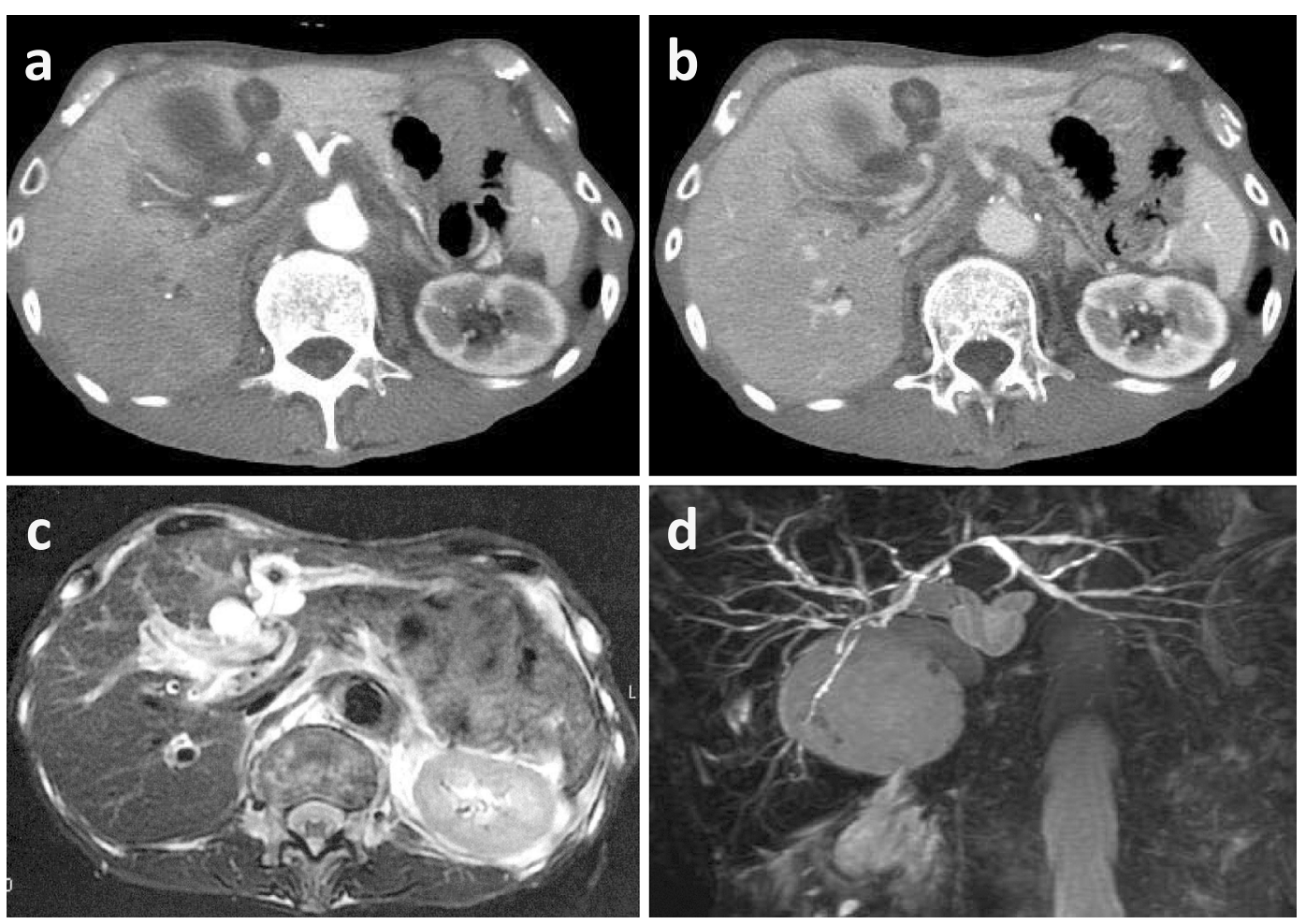

Figure 1. (a, b) Abdominal contrast-enhanced computed tomography (CE-CT): (a) early phase; (b) late phase. CE-CT showed slight enhancement of soft tissue surrounding the bile duct and areas adjacent to the celiac artery. (c) Abdominal MRI with T2-weighted imaging revealed a lesion of high signal intensity along the intrahepatic bile duct with a focus in the hepatic portal region. (d) MRCP demonstrated a slightly dilated intrahepatic bile duct and diffuse narrowing of the extrahepatic bile duct.

hydrate antigen $19-9=82 \mathrm{U} / \mathrm{mL}$.

\section{Imaging studies}

Abdominal ultrasonography revealed biliary sludge and swelling of the gallbladder, although no wall thickness or polypoid lesions were observed. Abdominal contrastenhanced computed tomography (CT) showed slight enhancement of soft tissue surrounding the bile duct and areas adjacent to the celiac artery (Fig. 1a, b), with lymphadenopathy in the abdominal cavity. Chest CT did not show any significant findings. Abdominal MRI with T2-weighted imaging showed a lesion of high signal intensity along the intrahepatic bile duct with a focus in the hepatic portal region (Fig. 1c). Magnetic resonance cholangiopancreatography (MRCP) also demonstrated a slightly dilated intrahepatic bile duct and diffuse narrowing of the extrahepatic bile duct (Fig. 1d).

\section{Clinical course}

A diagnosis of bile duct carcinoma was suspected based on the above-mentioned findings, although the features observed on the imaging studies were atypical. The dilatation of the intrahepatic bile duct was relatively mild compared to the degree of extrahepatic bile duct stenosis. Therefore, hematological disorders, including malignant lymphoma, were included in the differential diagnosis. Percutaneous transhe- patic gallbladder drainage was initially performed to reduce the patient's symptoms, which revealed mucus in the gallbladder. Percutaneous transhepatic biliary drainage was also performed, as the intrahepatic bile duct exhibited sufficient dilatation for needle insertion; however, both procedures failed to improve the patient's jaundice, and instead his condition progressed. Bile cytology of specimens obtained using both procedures were negative. Dyspnea and a decrease in the blood oxygen level were observed, and the patient's condition further deteriorated the day before his death. He died on the 16th hospital day due to rapid progression of the disease. An autopsy was performed after obtaining consent from his family.

\section{Autopsy}

At autopsy, the macroscopic findings were significant for Glisson's capsule expansion in the hepatic portal tract, and the patient's gallbladder was fully packed with mucus, although the mucosal surface of the gallbladder was smooth. There was congestion in both lungs; however, no thrombi were detected in the hilum of the pulmonary artery. Microscopically, infiltration of SRCC was observed surrounding the intrahepatic and extrahepatic bile ducts, although the tumor itself had not infiltrated the bile duct mucosa (Fig. 2a, b). In addition, infiltration of signet ring cells was noted in the lymphatic vessels. The proliferation of well- 

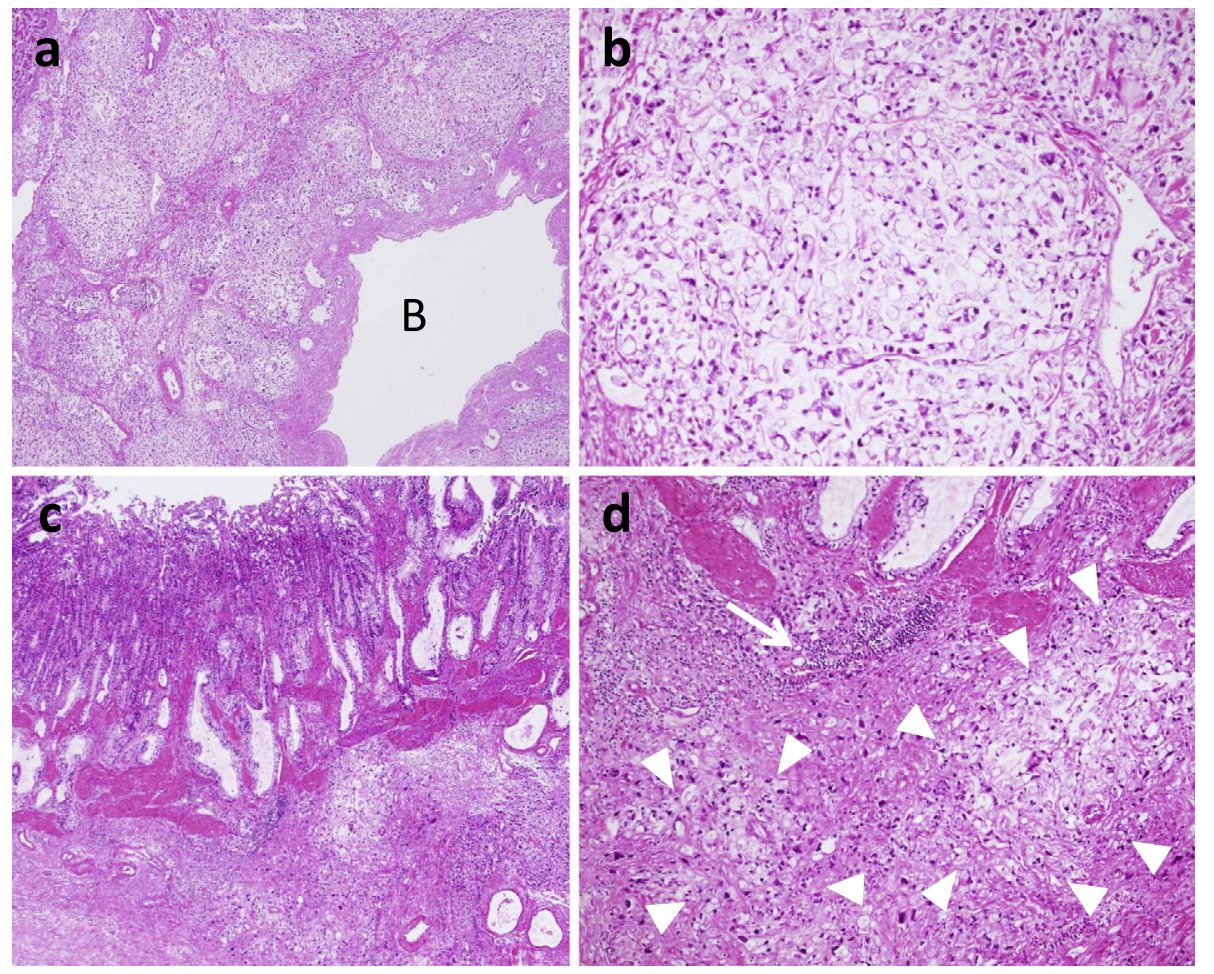

Figure 2. Histopathological findings. (a) Proliferation of SRCC around the bile duct (B), [Hematoxylin and Eosin (H\&E) staining, $\times 40$ ]. (b) SRCC (H\&E staining, $\times 200)$. (c, d) The gallbladder. (c) Proliferation of well-differentiated adenocarcinoma (H\&E staining, $\times 20)$, (d) destroying the muscle layer (arrow) and infiltration of SRCC into the subserosa (H\&E staining, $\times 200)$ (arrowheads).
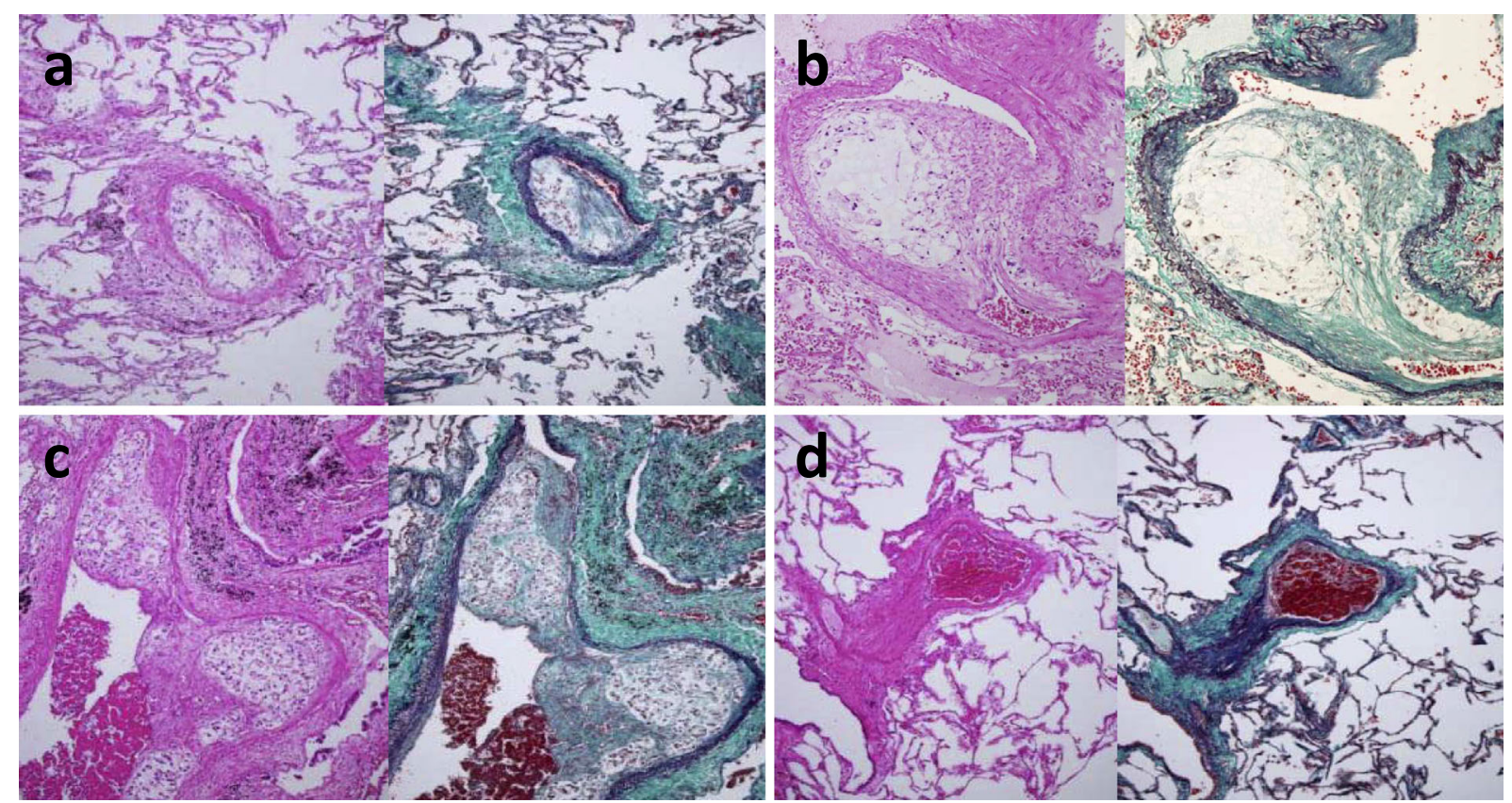

Figure 3. Histopathological findings of PTTM in the pulmonary arteries of the periphery of both lungs (left panels, Hematoxylin and Eosin staining; right panels, Elastica-Masson staining). (a, b) Fibrocellular intimal proliferation: (a) $300 \mu \mathrm{m}$ in diameter; (b) $500 \mu \mathrm{m}$ in diameter. (c) Tumor emboli. (d) Organized thrombi.

differentiated adenocarcinoma was observed in the gallbladder mucosa, infiltrating into the subserosa without growth toward the gallbladder lumen (Fig. 2c). Additionally, transi- tion from well-differentiated adenocarcinoma to SRCC was detected in the areas of infiltration (Fig. 2d). Lymph node metastasis, as well as direct invasion to the pancreas and 
duodenum, was also seen. Furthermore, fibrocellular intimal proliferation with the presence of tumor emboli and organized thrombi was observed in the periphery of the small pulmonary arteries with a diameter of 300-500 $\mu$ m (Fig. 3). The final diagnosis was SRCC of the gallbladder extending to the extra- and intrahepatic bile ducts and directly invading adjacent organs complicated by PTTM.

\section{Discussion}

We encountered a case of SRCC of the gallbladder complicated by PTTM that was diagnosed at autopsy. Adenocarcinoma is the most common histological type of gallbladder carcinoma, while SRCC is extremely rare. Nevertheless, conventional adenocarcinoma may occasionally be seen admixed with SRCC (7). SRCC can arise in virtually any organ, although most lesions originate from the stomach, breast or colon $(8,9)$. SRCC frequently metastasizes to peritoneal surfaces, as well as the regional lymph nodes, ovaries and lungs (9). In Japan, the incidence of SRCC has been reported to be approximately $1 \%$ of cases of primary gallbladder carcinoma diagnosed at autopsy (10). Minami et al. summarized 21 cases of SRCC of the gallbladder in Japan (11). Their results showed a slightly higher prevalence among women, with higher rates of cases admixed with other types of organized mucinous carcinoma, welldifferentiated adenocarcinoma and/or squamous cell carcinoma. In addition, many cases involving elevated lesions in the gallbladder lumen were observed. Many of the patients were diagnosed with SRCC of the gallbladder after undergoing cholecystectomy. The average survival rate was as short as 19 months.

Making the diagnosis was very difficult in the present case for two reasons. First, poor findings of gallbladder carcinoma were observed in the various imaging studies due to an unusual pattern of expansion; i.e., the tumor not growing into the gallbladder lumen, but rather infiltrating the subserosa. Second, the tumor exhibited poor expansion into the intrahepatic bile duct compared to the degree of common bile duct stenosis, and infiltration of SRCC was observed surrounding the intrahepatic and extrahepatic bile ducts.

In the present case, there was also marked intimal fibroplasia and medial hypertrophy involving small pulmonary arterioles consistent with a diagnosis of PTTM, which is characterized by the presence of multiple microthrombi and intimal myofibroblast proliferation (5). Patients with PTTM often present with rapidly progressive dyspnea and acute pulmonary hypertension (12). PTTM has been reported to be observed in $0.9-3.3 \%$ of cadavers with extrathoracic malignancies. Gastric adenocarcinoma is the malignancy most frequently associated with PTTM (5). Only two cases of PTTM arising from metastatic gallbladder carcinoma have been reported, one of which involved SRCC of the gallbladder $(6,13)$. Uruga et al. reported that, among 2,215 consecutive autopsy cases of carcinoma, 30 patients $(1.4 \%)$ were diagnosed with definitive PTTM (14). The median sur- vival time following the initiation of oxygen supplementation was nine days. There are some reports of the antemortem diagnosis of PTTM using pulmonary wedge aspiration cytology, lung biopsies, positron emission tomography or lung perfusion scans $(12,15-18)$. However, PTTM is generally difficult to diagnose prior to death and is primarily detected based on pathologic findings, as observed in the present case.

In summary, we herein reported a case of SRCC of the gallbladder associated with PTTM. It is important to recognize the origin of the primary tumor in order to optimize treatment. The proliferation of signet-ring cells in patients with gallbladder adenocarcinoma worsens the prognosis. A high level of clinical suspicion is required to make an antemortem diagnosis of PTTM.

The authors state that they have no Conflict of Interest (COI).

\section{References}

1. Bazan F, Sanchez J, Aguilar G, et al. Metastatic gallbladder adenocarcinoma with signet-ring cells. J Med Case Rep 5: 458, 2011.

2. Henson DE, Albores-Saavedra J, Corle D. Carcinoma of the gallbladder. Histologic types, stage of disease, grade, and survival rates. Cancer 70: 1493-1497, 1992.

3. Lazcano-Ponce EC, Miquel JF, Muñoz N, et al. Epidemiology and molecular pathology of gallbladder cancer. CA Cancer J Clin 51: 349-364, 2001.

4. Shields DJ, Edwards WD. Pulmonary hypertension attributable to neoplastic emboli: an autopsy study of 20 cases and a review of the literature. Cardiovasc Pathol 1: 279-287, 1992.

5. Von Herbay A, Illes A, Waldherr R, et al. Pulmonary tumor thrombotic microangiopathy with pulmonary hypertension. Cancer 66: 587-592, 1990.

6. Malani AK, Gupta C, Kutty AV, Betlej T. Pulmonary tumor thrombotic microangiopathy from metastatic gallbladder carcinoma: an unusual cause of severe pulmonary hypertension. Dig Dis Sci 52: 555-557, 2007.

7. Albores SJ, Eael HD, David SK. Tumors of the gallbladder, extrahepatic bile ducts, and ampulla of vater. In: Atlas of Tumor Pathology, Third Series, Fascicle 27. Armed Forces Institute of Pathology, Washington DC, 2000: 85-88.

8. Chu PG, Weiss LM. Immunohistochemical characterization of signet-ring cell carcinomas of the stomach, breast, and colon. Am J Clin Pathol 121: 884-892, 2004.

9. Pavić I, Marusić Z, Mijić A, et al. A case of signet-ring cell carcinoma of the gallbladder: immunohistochemistry and differential diagnosis. Acta Clin Croat 49: 159-162, 2010.

10. Annual of the Pathological Autopsy Cases in Japan 2008. The Japanese Society of Pathology (in Japanese).

11. Minami Y, Nagano $Y$, Ueda M, et al. A case of resected signet ring cell carcinoma arising from the gallbladder. Nihon Gekakei Gakkai Rengoukaishi (Journal of Japanese College of Surgeons) 34: 631-635, 2009 (in Japanese, Abstract in English).

12. Shih HM, Lin CC, Shiao YW. Pulmonary tumor thrombotic microangiopathy. Am J Emerg Med 29: 241.e3-241.e4, 2011.

13. de Luis DA, Darriba J, San Miguel P, et al. A case of secondary pulmonary hypertension due to microscopic pulmonary tumor cell embolism from gallbladder carcinoma. Respiration 64: 244-246, 1997.

14. Uruga H, Fujii T, Kurosaki A, et al. Pulmonary tumor thrombotic microangiopathy: a clinical analysis of 30 autopsy cases. Intern 
Med 52: 1317-1323, 2013.

15. Bhuvaneswaran JS, Venkitachalam CG, Sandhyamani S. Pulmonary wedge aspiration cytology in the diagnosis of recurrent tumour embolism causing pulmonary arterial hypertension. Int $\mathbf{J}$ Cardiol 39: 209-212, 1993.

16. Uruga H, Morokawa $\mathrm{N}$, Enomoto $\mathrm{T}$, et al. A case of pulmonary tumor thrombotic microangiopathy associated with lung adenocarcinoma diagnosed by CT-guided lung biopsy. Nihon Kokyuki
Gakkai Zasshi (Journal of the Japanese Respiratory Society) 46: 928-933, 2008 (in Japanese, Abstract in English).

17. Tashima $\mathrm{Y}$, Abe $\mathrm{K}$, Matsuo $\mathrm{Y}$, et al. Pulmonary tumor thrombotic microangiopathy: FDG-PET/CT findings. Clin Nucl Med 34: 175177, 2009.

18. Keenan NG, Nicholson AG, Oldershaw PJ. Fatal acute pulmonary hypertension caused by pulmonary tumour thrombotic microangiopathy. Int J Cardiol 124: e11-e13, 2008.

(C) 2014 The Japanese Society of Internal Medicine http://www.naika.or.jp/imonline/index.html 